\title{
Diacronie
}

Studi di Storia Contemporanea

$N^{\circ} 19,3 \mid 2014$

Miscellaneo

\section{Camillo Robertini (a cura di), Questa terra è la mia terra. Storie del Veneto, del Salento e dell'America}

\section{Latina}

\section{Cristina Celani}

\section{(2) OpenEdition \\ Journals}

\section{Edizione digitale}

URL: http://journals.openedition.org/diacronie/1656

DOI: 10.4000/diacronie.1656

ISSN: 2038-0925

\section{Editore}

Association culturelle Diacronie

Notizia bibliografica digitale

Cristina Celani, «Camillo Robertini (a cura di), Questa terra è la mia terra. Storie del Veneto, del Salento e dell'America Latina », Diacronie [Online], No 19, 3 | 2014, documento 11, Messo online il 01 septembre 2014, consultato il 22 septembre 2020. URL : http://journals.openedition.org/diacronie/1656 ; DOI : https://doi.org/10.4000/diacronie.1656 


\title{
RECENSIONE:
}

\section{Camillo ROBERTINI (a cura di), Questa terra è la mia terra. Storie del Veneto, del Salento e dell'America Latina, Venezia, La Toletta edizioni, 2013, 129 pp.}

\author{
a cura di Cristina CELANI *
}

Un gruppo di studenti dell'Università Ca' Foscari di Venezia decide un giorno di scrivere di storie, storie vive, di donne e uomini che hanno vissuto, storie differenti per i più disparati scenari geografici e politici. Questo gruppo formatosi grazie a un call for papers lanciato dal progetto Contemporaneamente - antropologia e storia, e, utilizzando le risorse fornite dall'Università per finanziare le attività formative autogestite dagli studenti, ha dato avvio a un percorso che si è concluso con il volume qui presentato: Questa terra è la ma terra. Storie del Veneto, del Salento e dell'America Latina.

Il volume, curato da Camillo Robertini ${ }^{1}$ raccoglie in tutto sette saggi. Il nucleo pregnante è la trasmissione esperienziale che crea un ponte tra narratore, studioso e lettore. Il contesto di studi si pone come un luogo di interdisciplinarietà tra storia della contemporaneità, storia orale e ricerca di carattere etnografico e antropologico. Gli autori dei saggi hanno avuto percorsi di vita e di studi certamente diversi, ma il filo conduttore dei loro vissuti sono le esperienze all'estero (Europa, Asia, Africa, America), frutto di una generazione ormai globalizzata, come scrive Alessandro Casellato nella Postfazione ${ }^{2}$. Francesca Correr, studentessa di storia e in seguito di antropologia presso l'Università Ca' Foscari di Venezia, per esempio, si interessa dei movimenti sociali e

\footnotetext{
${ }^{1}$ Dottorando in Studi Storici presso l'Università di Firenze, laureatosi in storia prima a Perugia e, successivamente, a Venezia è attualmente impegnato in una ricerca sulla classe operaia argentina durante la dittatura militare (1973-1976) frutto di un primo programma di interviste a militanti rivoluzionari e sindacali realizzate durante il suo periodo di studio in Argentina 2 Camillo ROBERTINI (a cura di), Questa terra è la ma terra. Storie del Veneto, del Salento e dell'America Latina, Venezia, La Toletta edizioni, 2013, p. 127.
} 
delle varie dinamiche socio-economiche e politiche in Bolivia $^{3}$, ed ha preso in considerazione le giornate della guerra dell'acqua di Cochabamba raccontate da uno dei suoi protagonisti, il guerero del agua Marcelo Rojas.

Marco Goldin 4 si occupa di Martignano descrivendo il paesaggio rurale salentino e i suoi cambiamenti, sia quelli che ha subito, sia quelli che ha vissuto. Paolo Grassi5, apartire dai suoi studi sul campo, sviluppati a Città del Guatemala, ha prodotto il saggio Storia e attualità di una Zona Rossa. Stefano Pontiggia ${ }^{6}$ è autore, nel volume, di un saggio sulle memorie in tre atti corali di un gruppo di istriani vittime dell'esodo forzato. Chiara Sacchet ${ }^{7}$ qui scrive dell'esperienza di vita e dei ricordi partigiani di un’intellettuale: Clementina Merlin detta Tina. Silvia Segalla ${ }^{8}$ traccia la vita di Caterina, operaia al Lanificio Rossi, il Lanerossi di Schio nel vicentino.

Sette racconti di donne e uomini tenacemente attaccati a microcosmi assai particolari, il legame con i quali è per essi una forma di resistenza. Il fatto che come titolo della raccolta - quasi un filo della collana che li tiene tutti legati - sia stata individuata l'autobiografia di un vagabondo "provinciale" come Woody Guthrie è una scelta quanto mai indovinata. E suggestiva9.

L'opera vuole far affacciare il lettore al tema della «trasmissione di frammenti o tracce che compongono la memoria che deriva dalla viva esperienza (esperienza

\footnotetext{
3 Si veda CORRER, Francesca, PAOLLILO, Antonio, Bolivia. Evoluzioni economiche e nuove dinamiche geopolitiche, Reggio Calabria, Isthar Editrice, 2012.

4 Ha studiato Geografia a Padova, frequentando un anno Erasmus a Siviglia, e Agroecologia a Baeza in Spagna ed ora è iscritto al master Environnement Développement Territoires et Sociétés a Parigi; si interessa della questione rurale connessa all'etnoecologia e all'agroecologia per uno sviluppo alternativo e sostenibile.

5 Ha studiato Scienze dell'Educazione, poi Scienze Antropologiche ed Etnologiche presso l'Università di Milano Bicocca. Successivamente ha frequentato il master in Analisi e Gestione di Progetti di Sviluppo dell'Università di Milano. È attualmente dottorando in Antropologia all'Università di Verona; di recente ha compiuto ricerche sui processi di segregazione urbana, questioni di violenze e potere, banditismo giovanile transnazionale. Sta sviluppando le sue ricerche a Città di Guatemala.

${ }^{6}$ Ha compiuto studi in Scienze dell'Educazione e Scienze antropologiche ed etnologiche presso l'Università di Milano Bicocca. È ora dottorando in Studi umanistici e sociali all'Università di Ferrara. I suoi temi principali di interesse sono l'intreccio fra memoria individuale e collettiva, memoria della violenza e ricomposizione sociale, memoria coloniale, politiche della memoria e costruzione nazionale.

7 Si è laureata in Filologia e letteratura italiana all'Università di Venezia. Si interessa da sempre di letture che si confondono con la storia, storie al femminile, vissute e raccontate.

$8 \mathrm{Si}$ è laureata in Lettere e in seguito in Interculturalità e Cittadinanza Sociale nell'ateneo veneziano con una tesi di ricerca sul lavoro femminile nell'industria vicentina. Attualmente è dottoranda dell'Università di Padova in Interazioni, Comunicazione e Costruzioni Culturali.

9 CASELLATO, Alessandro, «Postfazione», in ROBERTINI, Camillo (a cura di), op. cit., pp. 127128.
} 
sensibile) di donne e uomini che hanno vissuto ${ }^{10}$. Si vogliono condividere e divulgare esperienze lontane, estranee o distanti nel tempo e lontane per luoghi. Esperienze la cui trasmissione potrebbe interrompersi o così lontane da poter cadere nell'oblio. Il lettore non deve essere un addetto ai lavori; al contrario le varie storie hanno forma dialogica: gli autori, dando forma alle parole per preservarle e consegnarle al lettore, fanno spazio ai soggetti dei saggi attraverso interviste, verbali dei processi, memorie scritte. La raccolta ha carattere sperimentale, vuole essere un punto di partenza per ricerche successive. "Questo piccolo "libro primo", intreccio di storie, di luoghi e di generazioni, ci lascia con l'idea che la ricerca sociale, la scrittura, la stessa università non siano in fondo che l'esperienza di un incontro con mani inattese che ti scuotono, o ti sostengono, o ti consegnano qualcosa» ${ }^{11}$. Il gruppo di studenti di Ca' Foscari si pone interrogativi che nel campo delle scienze umane sono state analizzate in Europa o in America solo negli ultimi decenni: questioni di storie di vita, memoria culturale, memoria biografica. All'interno del dibattito interdisciplinare in corso (specialmente antropologia, storia orale e sociale), legato alla memoria culturale, esperti di differenti settori disciplinari affrontano temi come la soggettività, le emozioni, il contesto, il tempo e l'evoluzione, la tensione tra memoria e oblio, l'informazione e, infine, la memoria come costruzione identitaria.

La disomogeneità cosciente dei saggi, effetto del fatto che il libro è nato senza aver steso una traccia iniziale o senza seguire un concordato tema monografico, ha rivelato un tema comune: la terra, terra della nascita, della conciliazione, della pace, del rifiuto, del cambiamento. Il volume di racconti così intrecciati concettualmente prende spunto dall'omonimo libro scritto da Woody Guthrie ${ }^{12}$. Così come la vita da peregrino nell'America della Grande Depressione del cantante folk è segnata dalla terra, così lo è ogni esistenza dei personaggi dei racconti o della nostra.

La terra è quella che sembra quasi assumere i connotati di un paesaggio da favola della contadina-operaia Caterina, che vede sviluppare la sua vita intorno al lanificio "Lanerossi" di Piovene Rocchette, nel vicentino, prima del boom economico, fenomeno che stravolgerà il panorama urbano, umano e geografico. La terra è quella familiare e orfana del "popolo dell'esodo", che fu costretto al sacrificio di abbandonare l'Istria, divenuta ostile sotto il controllo jugoslavo, tra la fine degli anni Quaranta e gli anni Cinquanta, e costretto alla ricerca mai acquietata di un'identità ormai ideale nel territorio italiano. La terra è quella della piccola proprietà di Santa Tecla, in La casa

${ }^{10}$ ROBERTINI, Camillo, Introduzione, in ROBERTINI, Camillo (a cura di), op. cit., p. 7.

${ }^{11}$ CASELLATO, Alessandro, Postfazione, in ROBERTINI, Camillo (a cura di), op. cit., p. 129.

${ }^{12}$ GUTHRIE, Woody, Questa è la mia terra, Milano, Marcos y Marcos, 1997. 
sulla Marteniga, l'autobiografia di Tina Merlin: la guerra ha portato miseria, morti e orrori difficili da cancellare dal ricordo, allo stesso tempo bisogna dare giustizia a quanto è passato in onore del "dovere di parola e di memoria".

La terra è quella dell'Arneo, dove nel 28 Dicembre 1950 intorno alla tenuta Carignano si radunarono i contadini salentini con l'intento di occuparla, chiedendo di includere nella riforma agraria - che veniva varata in quel periodo - anche quel latifondo. A prendere parola nel racconto è perlopiù Giuseppe Armando Meleleo. Dopo il processo, che pure si concluse con un verdetto a favore dei contadini, questi non ebbero un pezzo di quella terra tanto desiderata e furono costretti ad emigrare in Belgio e in Svizzera. Qui emerge un ulteriore aspetto importante: il mancato riconoscimento da parte delle generazioni successive; questi ultimi sono protagonisti dell'abbandono di quelle terre per le quali i propri padri e nonni avevano combattuto strenuamente con la lotta. La terra è, ancora una volta, quella salentina di Martignano, dove inique politiche hanno piegato l'agricoltura di questi posti vietando la produzione di tabacco in nome di un modello di produzione urbano, costringendo così la popolazione a emigrare dalle proprie terre natie inaridite nella loro funzione di sostentamento che avevano un tempo.

La terra è la terra boliviana della città di Cochabamba, dove nel 2000 si verificarono scontri per la "guerra dell'acqua”, narrata da Marcelo Rojas, il sindacalista definito guerero del agua. La lotta reclamava una politica per il proprio paese che potesse rispondere a istanze ecologiste, rivendicazioni etniche e sindacaliste. La terra è anche quella guatemaleca dove chi non aveva una terra occupò il quartiere del barrio a Città del Guatemala. Non è stata dedicata molta attenzione al fenomeno, gli abitanti del quartiere pur di avere a disposizione una terra propria, accettarono una condizione di provvisorietà e informalità. Quando tale spazio fu ufficializzato venne contestualmente etichettato come "zona rossa", piena di criminali, bande e caratterizzata da una povertà diffusa.

L'esperienza degli studenti e autori dei racconti è stata vissuta con una ferma convinzione: la terra è la chiave interpretativa di ogni esistenza. La parola si ripete molte volte all'interno del libro, avvicinando tutti e sette i racconti. È la terra a muovere le persone, compresi i fenomeni migratori, come nel caso dell'esodo istriano. È la terra che muove anche guerre e conflitti, come nel contesto latino-americano dove si lotta per salvaguardare beni sociali comuni, come l'acqua o la casa.

Le storie sono frutto di ricerche sul campo che hanno portato ad interagire in modo diretto con i protagonisti, per cercare di cogliere tutti gli aspetti storici con la convinzione che terra e vita siano inevitabilmente intrecciati. Tutti gli aspetti colti, una 
volta messi insieme, fanno emergere uno "scenario geografico, politico e diacronico" frastagliato, che rimane coerente con la volontà degli autori di evidenziare quanto $\mathrm{i}$ fenomeni storici possano essere frammentari e incoerenti. È naturale ora credere che è proprio da qui che si vuole partire, ossia dal racconto narrato, dall'interazione sociale, dalle relazioni informali, da «storie normali»13.

Si tratta [...] di un tentativo molto più concreto: quello di recuperare il filo interrotto tra chi ha da raccontare una storia e chi, per qualsiasi motivo, non abbia avuto la fortuna di conoscere Tina Merlin, Marcelo Rojas, Giuseppe Meleleo o Caterina ${ }^{14}$.

L'opera ha in generale una sua struttura coerente, che si basa sul ruolo della narrazione e della ricerca umana. Sarebbe difficile fare antropologia senza che questa divenga, per necessità, anche una scienza storica il cui interesse si concentra sul tentativo di comprendere i fenomeni individuali piuttosto che sulla definizione di leggi generali, che potrebbero sembrare vaghe. Nel volume il problema della terra è declinato in vari modi e funge da sfondo ai racconti che mantengono in ogni modo una loro indipendenza stilistica e tematica. Gli autori hanno voluto catturare l'attenzione del lettore in modo tale che egli potesse immedesimarsi e condividere con i protagonisti delle storie quanto da questi provato. Solo così il lettore può viaggiare nel tempo e nello spazio per vivere stralci di vita che non avrebbe mai potuto vivere, interrogandosi su quanto accaduto.

Accanto al tema della terra, quindi, il tema della memoria risulta di fondamentale importanza. Per ricostruire il passato, più che parlare di memorie, si può parlare di "fare" memoria, mettendo in una nuova prospettiva eventi, interpretazioni e significati. La memoria, pertanto, deve essere trattata per ciò che è: un campo di pratiche. Raccontare memorie rende le persone portavoce di racconti, nello spazio pubblico e privato. Come ha fatto Tina Merlin, la quale «conferisce una dimensione quotidiana ai giorni della Resistenza, e pone un presidio permanente alla difesa della memoria e delle memorie, rifiutando sempre una facile acquiescenza e una riconciliazione postuma» ${ }^{15}$, è nell'apparente quiete dei giorni che va ricercato il filo del racconto, il dovere della parola e della memoria, in onore del passato e del futuro. Come scrisse Tina Merlin, infatti, il contrario della memoria non è l'oblio, ma la giustizia.

${ }^{13}$ ROBERTINI, Camillo, Introduzione, in ROBERTINI, Camillo (a cura di), op. cit., p. 9.

14 Ibidem.

${ }^{15}$ SACCHET, Chiara, Tina Merlin. Che il contrario di oblio non sia memoria, ma giustizia, in ROBERTINI, Camillo (a cura di), op. cit., p. 51. 


\section{* L'autore}

Cristina Celani ha conseguito nel 2011 il diploma di laurea triennale in Filosofia presso l'Università di Bologna. Attualmente è studente del corso di laurea magistrale in Scienze Filosofiche nello stesso ateneo. I suoi studi vertono principalmente sulla storia della psicologia, sulla filosofia morale e sulla filosofia del diritto.

URL: < http://www.studistorici.com/2013/10/o5/cristina-celani/ >

\section{Per citare questo articolo:}

CELANI, Cristina, «Recensione: Camillo ROBERTINI (a cura di), Questa terra è la mia terra. Storie del Veneto, del Salento e dell'America Latina, Venezia, La Toletta edizioni, 2013, 129 pp.», Diacronie. Studi di Storia Contemporanea: , 29/09/2012,

URL:<http://www.studistorici.com/2014/09/29/celani_numero_19/ >

\section{Diacronie Studi di Storia Contemporanea $\beta$ www.diacronie.it}

Risorsa digitale indipendente a carattere storiografico. Uscita trimestrale. redazione.diacronie@hotmail.it

Comitato di redazione: Jacopo Bassi - Luca Bufarale - Elisa Grandi - Deborah Paci - Fausto Pietrancosta - Matteo Tomasoni - Luca Zuccolo

CC (i) $\odot$ Diritti: gli articoli di Diacronie. Studi di Storia Contemporanea sono pubblicati sotto licenza Creative Commons 2.5 . cc Possono essere riprodotti a patto di non modificarne i contenuti e di non usarli per fini commerciali. La citazione di BY NC ND estratti è comunque sempre autorizzata, nei limiti previsti dalla legge. 